\title{
OFCDM: A Promising Broadband Wireless Access Technique
}

\author{
Yiqing Zhou and Tung-Sang Ng, University of Hong Kong \\ Jiangzhou Wang, University of Kent \\ Kenichi Higuchi and Mamoru Sawahashi, NTT DoCoMo
}

\begin{abstract}
Future mobile communication systems aim to provide extremely high-speed data transmission, especially in the downlink. The broadband orthogonal frequency- and code-division multiplexing system with two-dimensional spreading (time and frequency domain spreading) is becoming a very promising technique for highspeed wireless communications due to its advantages over OFDM. This article presents the basic structure and main functions of the OFCDM system. A nonsequential code assignment scheme is introduced. The novel detection method for the OFCDM, called hybrid multicode interference cancellation and minimum mean square error detection, is described. The application of advanced techniques to the OFCDM, such as turbo coding and MIMO, is also discussed. It is shown in this article that OFCDM is superior to OFDM.
\end{abstract}

\section{INTRODUCTION}

The era of high-speed data transmission in mobile communications is approaching rapidly. Wideband code-division multiple access (WCDMA), one of the third-generation (3G) wireless networks, was launched first by NTT DoCoMo, Japan, in 2001. With a bandwidth of 5 $\mathrm{MHz}$, a WCDMA system can provide multimedia services with data rates up to $2 \mathrm{Mb} / \mathrm{s}$. In addition, high-speed downlink packet access (HSDPA) has been introduced in the WCDMA system by using advanced techniques, such as adaptive modulation and coding and hybrid automatic repeat request (ARQ), and the data rate can be increased to $14 \mathrm{Mb} / \mathrm{s}$. With high data rates, subscribers of the WCDMA network can get richer multimedia services. However, the data rate requirement increases dramatically due to demand for high-speed multimedia services. In the 2010s, the expected wireless data rate could be up to several gigabits per second, which is far beyond the data rate provided by current $3 \mathrm{G}$ and other wireless systems. The future fourth-generation $(4 \mathrm{G})$ mobile communication systems are designed to fill this big gap. The research target of $4 \mathrm{G}$ is to achieve $100 \mathrm{Mb} / \mathrm{s}$ peak data rate for wide area coverage with full mobility and $1 \mathrm{~Gb} / \mathrm{s}$ peak data rate for local area coverage with low mobility. The $4 \mathrm{G}$ systems will support multimedia services like high-speed Internet access and broadcast services from information sites. Due to the nature of these multimedia services, the data traffic in the downlink is expected to be much more than that in the uplink. Therefore, high data rates are especially necessary for $4 \mathrm{G}$ in the downlink. Although the maximum bandwidth of $3 \mathrm{G}$ long-term evolution can be up to $20 \mathrm{MHz}$ for a peak rate of 100 $\mathrm{Mb} / \mathrm{s}$, the bandwidth of the future $4 \mathrm{G}$ system should be much larger than $20 \mathrm{MHz}$ to transmit a peak rate of $1 \mathrm{~Gb} / \mathrm{s}$. Therefore, a new wireless access technique using 50-100 MHz bandwidth is needed for wireless transmission in the downlink of $4 \mathrm{G}$ systems.

Various wireless access schemes have been proposed for the broadband downlink transmission in $4 \mathrm{G}$ systems. Single-carrier CDMA is not suitable over a broadband channel due to too much multipath interference (MPI) [1]. On the other hand, a multicarrier approach, orthogonal frequency-division multiplexing (OFDM), has drawn a lot of attention in high-speed wireless communications. OFDM employs a large number of orthogonal subcarriers to transmit symbols in parallel, so the symbol duration is increased substantially and the system can combat MPI. Since the modulation/demodulation of a large number of subcarriers can be realized by inverse fast Fourier transform (IFFT)/FFT, OFDM is easy to implement and cost effective. Orthogonal frequency-domain multiple access (OFDMA) can provide multiple access by addressing a subset of subcarriers to individual receivers. Moreover, it readily supports narrowband interference suppression and multiple antenna techniques to enhance efficiency. Due to all these advantages, OFDM has been chosen as the transmission method for various standards such as the European radio (DAB) and TV (DVB-T) standard, IEEE 802.11 standards for wireless LANs, and IEEE 802.16 standards for wireless MANs.

Although OFDM is attractive for high-speed 


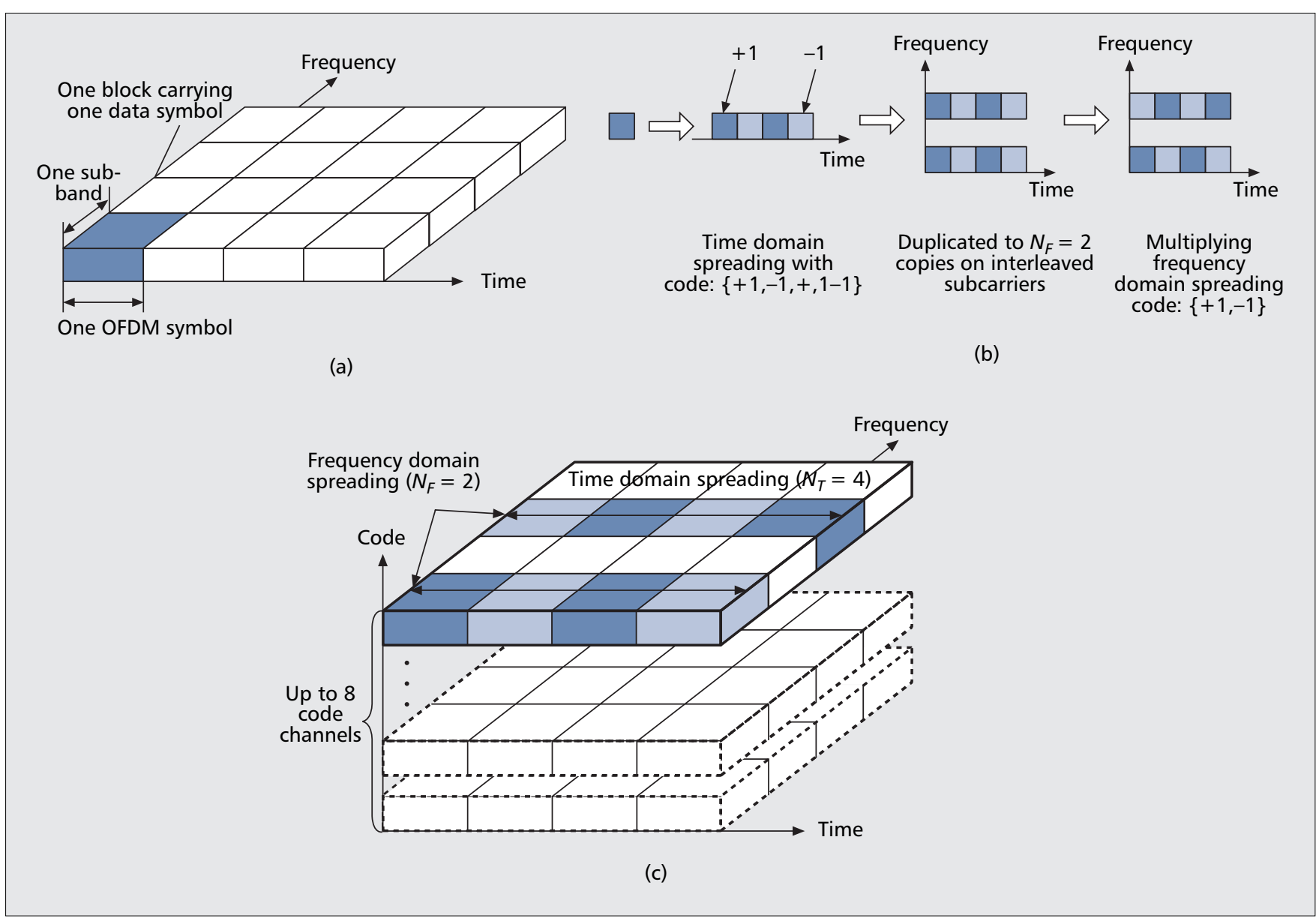

Figure 1. Difference between OFDM and OFCDM: a) OFDM without spreading; $b$ ) 2D spreading; c) OFCDM with 2D spreading.

wireless communications, it does not have coherent frequency diversity. Moreover, in mobile cellular systems, OFDM suffers from adjacent cell interference unless the same subcarriers are not used among adjacent cells. Thus, spreading has been introduced to OFDM to provide frequency diversity and facilitate one-cell frequency reuse in a cellular environment. Combining OFDM with two-dimensional spreading (time and frequency domain spreading), an orthogonal frequency- and code-division multiplexing (OFCDM) system has been proposed for the downlink transmission in future $4 \mathrm{G}$ networks [2]. Based on OFDM, OFCDM provides not only all advantages of OFDM, but also additional benefits by means of 2D spreading. For example, frequency diversity gain can be achieved through frequency domain despreading due to the different fading experienced by subcarriers in a broadband channel. Furthermore, with the introduction of time domain spreading, the system can provide flexible transmission rates. The time and frequency domain spreading factors $N_{T}$ and $N_{F}$ can be changed flexibly to provide variable spreading factor (VSF) in order for the system to work in different cell environments and channel conditions. The effectiveness of OFCDM has been tested by NTT DoCoMo in field experiments [3]. By using OFCDM and a bandwidth of $100 \mathrm{MHz}$, a data transmission rate of $100 \mathrm{Mb} / \mathrm{s}$ in the downlink has been achieved without the use of multiple-input multiple-out- put (MIMO) in outdoor environments at a moving speed of $20 \mathrm{~km} / \mathrm{h}$. Thus, OFCDM has proven to be a promising candidate for downlink access in future $4 \mathrm{G}$ mobile networks. This article focuses on high speed transmission using OFCDM with low mobility. When the moving speed of a user is high, transmission data rate must be slow.

\section{OFDM VS. OFCDM}

The basic difference between OFDM and OFCDM is illustrated in Fig. 1. In OFDM (Fig. 1a), the radio resource is divided into frequencytime blocks. Each block occupies one OFDM symbol duration in the time domain and one subcarrier in the frequency domain. Since one data symbol is conveyed by each frequency-time block, the OFDM system in Fig. 1a can send a total of 16 data symbols on four subcarriers in four OFDM symbol durations. Figure 1b illustrates simple $2 \mathrm{D}$ spreading on one data code channel using a spreading factor of $N=N_{T} \times N_{F}$ $=4 \times 2$, where $N$ is the total spreading factor. It can be seen that in 2D spreading, each data symbol is first spread by the time domain spreading code with $\{+1,-1,+1,-1\}$, and $N_{T}=4$ chips are obtained. Then the time domain spread signal is duplicated into $N_{F}=2$ copies in the frequency domain and multiplied by the frequency domain spreading code $\{+1,-1\}$. Note that in order to achieve good frequency diversity gain, $N_{F}$ interleaved subcarriers are employed instead 
Up to eight 2-D

code channels are

available in the

OFCDM system,

which can provide

various service rates

by assigning different

number of code

channels to one

single user. When all

the eight code

channels are

employed, OFCDM

can achieve the

same data rate as

that in OFDM.

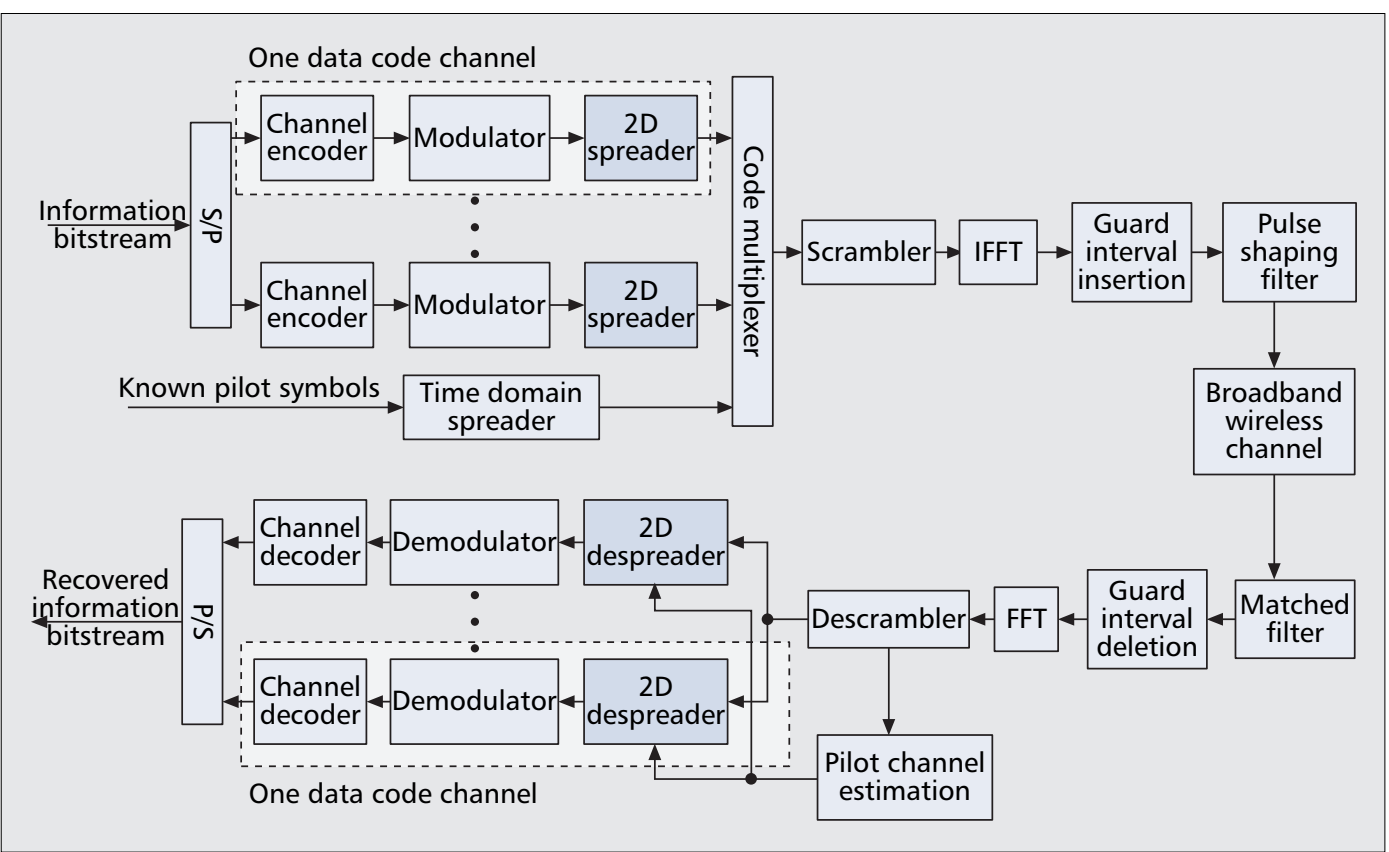

Figure 2. Block diagram of the OFCDM system. of $N_{F}$ consecutive subcarriers. For example, with a block interleaver and a total number of 4 subcarriers, the first copy of the $N_{T}$ chips is impressed on the 0th subcarrier and the second copy is impressed on the second subcarrier.

Combining OFDM with 2D spreading, the resultant OFCDM is shown in Fig. 1c. It can be seen that on the dedicated $2 \mathrm{D}$ code channel with time domain spreading code $\{+1,-1,+1,-1\}$ and frequency domain spreading code $\{+1,-1\}$, one data symbol is transmitted on two interleaved subcarriers in four continuous OFCDM symbol durations. The 2D spreading in OFCDM is different from the conventional spreading in CDMA, which expands the signal bandwidth. Instead, it is more like a coding scheme carrying the same data information in $N=N_{T} \times N_{F}$ frequency-time blocks. Thus, redundancy is introduced, and the information data rate is decreased. In Fig. 1c each code channel can only convey two data symbols on four subcarriers in four OFCDM symbol durations, resulting in a data rate $1 / N$ of that in OFDM.

To increase the data rate, a multicode transmission technique can be employed. Orthogonal variable spreading factor (OVSF) codes [4] are used as the spreading codes in both the frequency and time domains. As shown in Fig. 1c, there are two and four different spreading codes in frequency and time domains, respectively. Hence, up to eight 2D code channels are available in the OFCDM system, which can provide various service rates by assigning different numbers of code channels to a single user. When all eight code channels are employed, OFCDM can achieve the same data rate as that in OFDM. In fact, OFDM can be considered a special case of OFCDM with a spreading factor of $1=1 \times 1$.

Generally speaking, given a $2 \mathrm{D}$ spreading factor of $N=N_{T} \times N_{F}$ and total $K \leq N$ code channels, the $k$ th code channel in the OFCDM system employs a $2 \mathrm{D}$ code $\left\{C_{N T}^{(k T)}, C_{N F}^{(k F)}\right\}$, where $k_{F} \in[0$,
$\left.N_{F}-1\right]$ and $k_{T} \in\left[0, N_{T}-1\right]$. Then the code set of the other $K-1$ code channels can be divided into two subsets: one set, $\Omega_{F}$, with the same time domain spreading code as the $k$ th code but different frequency domain spreading codes,

$$
\Omega_{F}=\left\{C_{N_{T}}^{\left(k_{T}\right)}, C_{N_{F}}^{\left(\bar{k}_{F}\right)}\right\},
$$

where $\bar{k}_{F} \neq k_{F}$; and the other set, $\Omega_{T}$, with different time domain spreading codes,

$$
\Omega_{T}=\left\{C_{N_{T}}^{\left(\bar{k}_{T}\right)}, C_{N_{F}}^{\left(\bar{k}_{F}\right)}\right\},
$$

where $\bar{k}_{T} \neq k_{T}$, but $\bar{k}_{F}$ can be any value in $\left[0, N_{F}\right.$ - 1], including $k_{F}$. Using OVSF codes, there would be no interference among the $K$ multiple code channels in an additive white Gaussian noise (AWGN) or a static frequency flat fading channel, according to the orthogonality of codes in frequency or time domain spreading. However, in a realistic mobile channel, the orthogonality between any two code channels may be destroyed by possible fast fading in the time domain or different fading among subcarriers in the frequency domain, and multicode interference (MCI) may occur. Combating MCI is the main concern of the OFCDM system.

\section{SYSTEM CONFIGURATION}

A block diagram of an OFCDM system [5] is shown in Fig. 2. At the transmitter, information data streams are first serial-to-parallel converted into multiple streams to be transmitted on multiple code channels. On each data code channel, information bits are processed by a channel encoder and modulated. Each modulated data symbol will be $2 \mathrm{D}$ spread by a dedicated $2 \mathrm{D}$ code for the data channel. In order to obtain good channel estimation, a code-multiplexed pilot channel is employed. However, the OFCDM system can support either a frequency-, 


\begin{tabular}{|c|c|c|}
\hline System parameters & OFDM & OFCDM \\
\hline Bandwidth & \multicolumn{2}{|c|}{$100 \mathrm{MHz}$} \\
\hline Number of subcarriers & \multicolumn{2}{|c|}{1024} \\
\hline Subcarrier spacing & \multicolumn{2}{|c|}{$97.7 \mathrm{kHz}$} \\
\hline OFDM/OFCDM symbol duration & \multicolumn{2}{|c|}{12.5 us (10.24 + 2.26: effective symbol + guard interval) } \\
\hline Packet length & \multicolumn{2}{|c|}{$0.6 \mathrm{~ms}(48 \times 12.5 \mu \mathrm{s}): 48$ OFDM/OFCDM symbols } \\
\hline Channel coding & \multicolumn{2}{|c|}{ Turbo codes } \\
\hline Modulation & \multicolumn{2}{|c|}{ QPSK } \\
\hline Spreading schemes & Without spreading & 2D spreading \\
\hline Pilot channel & $\begin{array}{l}\text { Time multiplexed, } N_{P} \text { pilot symbols } \\
\text { at the start of each packet }\end{array}$ & $\begin{array}{l}\text { Code multiplexed, time domain } \\
\text { spread with a factor of } N_{T}\end{array}$ \\
\hline
\end{tabular}

Table 1. System parameters.

time-, or code-multiplexed pilot channel. Among the three schemes, the time- and code-multiplexed pilot channels provide similar channel estimation quality and outperform the frequency-multiplexed one. The code-multiplexed scheme is preferred since it provides more flexibility in system design than the time-multiplexed scheme. To keep the orthogonality between the pilot and data code channels in frequency selective but slow fading channels, known pilot symbols are spread only in the time domain with the spreading factor of $N_{T}$. So there are $N_{T}-1$ different time domain spreading codes for data transmission. Then all code channels are combined at the code multiplexer. After code multiplexing, the combined signal is scrambled, upconverted to a set of subcarriers, and transmitted in parallel. An IFFT block realizes this upconversion operation. At the output of IFFT, an effective OFCDM symbol (or time chip) is obtained. A guard interval is inserted between effective symbols to prevent intersymbol interference (ISI). Finally, OFCDM symbols are input to the pulse shaping filter before transmission.

After passing through a broadband wireless channel, the received signals are first processed by a matched filter. Then the guard interval is removed, and the resultant signals are further processed by an FFT block, which realizes the subcarrier downconversion. After FFT, the signals are descrambled. On one hand, the output of the descrambler is used to carry out pilot channel estimation. On the other hand, the output of the descrambler is passed to the 2D despreader, where the estimated channel is needed. After 2D despreading, the obtained signals are demodulated and then decoded. At the output of the decoder, the transmitted information bits on each data code channel are recovered. It can be seen that basically, OFCDM is an OFDM system but with the addition of $2 \mathrm{D}$ spreading/despreading operation.

The system parameters for the OFCDM are summarized in Table 1. As a comparison, the parameters of OFDM are also shown. It can be seen that the basic radio link parameters of OFCDM and OFDM are the same. In the frequency domain, both systems employ a broad bandwidth of $100 \mathrm{MHz}$. Given the bandwidth, the larger the number of subcarriers, the longer the OFCDM symbol duration and thus the more robust the system to MPI. However, as the number of subcarriers increases, the subcarrier spacing decreases, which makes the system vulnerable to frequency shifts caused by Doppler shifts. Based on the optimization to compensate for the maximum multipath delay and avoid the influence of Doppler shift, the number of subcarriers $M$ is chosen to be 1024 , which results in a subcarrier spacing of $\Delta_{f}=97.7 \mathrm{kHz}$ and an effective OFCDM symbol length of $10.24 \mu \mathrm{s}$. In future $4 \mathrm{G}$ systems, since the information bit rate should be very high, a much smaller cell size than that of the current cellular system is inevitable considering the attainable transmission power. Thus, a microcell model with the cell radius of less than $1 \mathrm{~km}$ in an urban area is assumed. In such an environmentc the multipath delay spread should be much smaller. For example, the measured maximum multipath delay was approximately $1 \mu \mathrm{s}$ in field experiments conducted near downtown Tokyo with the distance between a base station and a mobile station of approximately $1 \mathrm{~km} \mathrm{[6].} \mathrm{Thus,} \mathrm{a} \mathrm{guard} \mathrm{interval} \mathrm{of}$ $2.26 \mu$ s would be sufficient to combat MPI. To reduce the round-trip delay, a short packet length of $0.6 \mathrm{~ms}$ is chosen, which is composed of $N_{d}=48$ OFCDM symbols. For channel coding, turbo codes are possible candidates. Quaternary phase shift keying (QPSK) modulation is employed. For the OFCDM system, 2D spreading is employed. A pilot channel is code-multiplexed with data channels by spreading in the time domain with a factor of $N_{T}$. On the other hand, in OFDM there is no spreading, and a timemultiplexed pilot channel is employed. $N_{P}$ pilot symbols are allocated at the start of each packet.

Given the
bandwidth, the
larger the number of
subcarriers, the
longer the OFCDM
symbol duration and
thus the more robust
of the system to
MPl. However, as
the number of
subcarriers
increases, the
subcarriers spacing
reduces, which
makes the system
vulnerable to
frequency shifts
caused by Doppler
shifts.




\section{Code Assigniment}

The variance of the

$\mathrm{MCl}$ generated

between any pair of code channels is

dependent on which codes to be used.

Use of some codes

for channel

spreading may result

in larger $\mathrm{MCl}$ than

that of others.

In order to improve

the system

performance, $\mathrm{MCl}$

should be cancelled

out as much as

possible.
As stated before, in a broadband channel all multi-code channels in the OFCDM system may interfere with each other and MCI is present due to possible fast fading in the time domain or different fading among subcarriers in the frequency domain. It is noted that the variance of the MCI generated between any pair of code channels is dependent on which codes are used. Use of some codes for channel spreading may result in larger MCI than others. In order to improve system performance, MCI should be canceled out as much as possible. This section discusses code assignment schemes to allocate appropriate codes for data transmissions so that the MCI on all code channels can be mitigated.

First of all, code channels in different subsets $\left(\Omega_{F}\right.$ or $\left.\Omega_{T}\right)$ have different contributions to the MCI on the $k$ th code channel. In highly frequency selective broadband channels, code channels from $\Omega_{F}$ cause severe MCI (MCI-F) to the $k$ th code channel because their orthogonality in the frequency domain is distorted by frequency selective fading on interleaved subcarriers. On the other hand, in fast fading channels code channels from $\Omega_{T}$ are no longer orthogonal to the $k$ th code channel in the time domain. Thus, there is also MCI from $\Omega_{T}$ (MCI-T). However, since a short packet length such as $0.6 \mathrm{~ms}$ is considered, the MCI-T is anticipated to be much lower than the MCI-F since the channel variation within one packet duration in the time domain is much less serious than that on interleaved subcarriers in the frequency domain. As there are totally $N_{T}-1$ time domain orthogonal codes for data transmission, when the number of required data code channels is less than or equal to $N_{T}-1$, MCI between any two code channels can be kept small by assigning channels with different time domain orthogonal codes. In this case $\Omega_{F}$ does not exist. However, when more than $N_{T}-1$ code channels are needed for data transmission, reuse of the same time domain spreading codes is unavoidable, and $\Omega_{F}$ must exist, so serious MCI-F occurs. To keep MCI small for each code channel, time domain spreading codes should be assigned first.

In slow fading channels, when assigning different codes within the time or frequency domain, there is no requirement on the order. The code assignment can be carried out sequentially as follows: $C_{N T}^{(0)}$ for the pilot channel, $\left\{C_{N T}^{(1)}, C_{N F}^{(0)}\right\}$ for the 0 th data code channel,

$$
\left\{C_{N_{T}}^{(2)}, C_{N_{F}}^{(0)}\right\}
$$

for the first data channel, ...,

$$
\left\{C_{N_{T}}^{\left(N_{T}-1\right)}, C_{N_{F}}^{(0)}\right\}
$$

for the $\left(N_{T}-2\right)$ th data channel,

$$
\left\{C_{N_{T}}^{(1)}, C_{N_{F}}^{(1)}\right\}
$$

for the $\left(N_{T}-1\right)$ th data channel, and so on. However, in fast fading channels, this simple sequential code assignment scheme is no longer suitable. For instance, after $C_{16}^{(0)}$ is assigned to the pilot channel, if $C_{16}^{(1)}$ or $C_{16}^{(15)}$ is allocated to the 0th data code channel, it has been shown [7] that the variance of the MCI-T generated between $C_{16}^{(0)}$ and $C_{16}^{(1)}$ is much higher than that between $C_{16}^{(0)}$ and $C_{16}^{(15)}$. Obviously, to keep the MCI-T small, $C_{16}^{(15)}$ should be assigned to the 0th data code channel instead of $C_{16}^{(19)}$. Therefore, a nonsequential code assignment scheme is needed to reduce MCI in fast fading channels.

After investigating the variance of MCI, it has been shown that the code assignment schemes obtained by minimizing MCI can be interpreted using code distances. The distance of two OVSF codes $C_{N T}^{\left(k_{0}\right)}$ and $C_{N T}^{\left(k_{1}\right)}$ is defined as $N_{T} / 2+1$ subtracted by the minimum length of strings of consecutive $1 \mathrm{~s}$ or $-1 \mathrm{~s}$ in the elementwise code product,

$$
C_{N_{T}}^{\left(k_{0}\right)} \cdot C_{N_{T}}^{\left(k_{1}\right)}=\left\{c_{N_{T}, 0}^{\left(k_{0}\right)} \cdot c_{N_{T}, 0}^{\left(k_{1}\right)}, \ldots, c_{N_{T}, N_{T-1}}^{\left(k_{0}\right)} \cdot c_{N_{T}, N_{T}-1}^{\left(k_{1}\right)}\right\} .
$$

For example, consider the distance between $C_{16}^{(0)}$ and $C_{16}^{(1)}$. Since $C_{16}^{(0)}$ is the all +1 code, the code product $C_{16}^{(0)} \cdot C_{16}^{(1)}$ equals $C_{16}^{(1)}$. There are two equal strings of eight consecutive $1 \mathrm{~s}$ and eight consecutive $-1 \mathrm{~s}$ in $C_{16}^{(1)}$. Therefore, the distance is one $(9-8=1)$. It is noted that MCIs are dependant on the code distance. The smaller the code distance, the larger the MCIs. Therefore, the codes with large code distances to each other should be assigned with high priority.

Moreover, in time domain code assignment, special consideration should be made of the MCI-T caused by the pilot channel since it has higher power than that on a data channel. Since $C_{N T}^{(0)}$ is assigned to the pilot channel, the code with a minimum distance to the pilot code is always $C_{N T}^{(1)}$. $C_{N T}^{(1)}$ suffers from much more interference from the pilot than other codes due to the shorter code distance. On the other hand, the MCI-T also depends on the channel variation in the time domain or the Doppler shifts. Given the same Doppler shift, the channel variation in an $N_{T}$ OFCDM symbol duration increases as $N_{T}$ increases, which results in a higher MCI-T for a larger $N_{T}$. In summary, in order to avoid large pilot interference, $C_{N T}^{(1)}$ is suggested not to be used for data transmission when $N_{T}$ is large, although it can be used when $N_{T}$ is small (i.e., $N_{T}=4$ or 8 ). The complete nonsequential code assignment schemes in time and frequency domains are shown in Table 2. It has been shown [7] that in fast fading channels, system performance can be improved significantly by the nonsequential scheme, especially for a low to medium system load where a small number of code channels are needed. For a highly loaded system, performance improvement is limited. When a fully loaded system is considered, there is no need for code assignment since all code channels must be assigned for data transmissions.

\section{HYBRID DETECTION}

Although nonsequential code assignment schemes help reduce MCI, at the receive side of the OFCDM, a powerful and efficient signal detection algorithm is needed in the 2D despreader to cancel out MCI as much as possible, especially for highly loaded systems. As 
Time domain spreading codes $\left(C_{N T}^{(1)}\right.$ is not assigned when $\left.N_{T}=16\right)$

\begin{tabular}{llllllllllllllll}
\hline $\begin{array}{l}\text { Assignment } \\
\text { order }\end{array}$ & $\begin{array}{l}1 \text { (for } \\
\text { pilot) }\end{array}$ & 2 & 3 & 4 & 5 & 6 & 7 & 8 & 9 & 10 & 11 & 12 & 13 & 14 & 15 \\
\hline$N_{T}=4$ & $C_{4}^{(0)}$ & $C_{4}^{(3)}$ & $C_{4}^{(2)}$ & $C_{4}^{(1)}$ & & & & & & & & \\
$N_{T}=8$ & $C_{8}^{(0)}$ & $C_{8}^{(7)}$ & $C_{8}^{(3)}$ & $C_{8}^{(4)}$ & $C_{8}^{(6)}$ & $C_{8}^{(5)}$ & $C_{8}^{(2)}$ & $C_{8}^{(1)}$ & & & & & \\
$N_{T}=16$ & $C_{16}^{(0)}$ & $C_{16}^{(15)}$ & $C_{16}^{(6)}$ & $C_{16}^{(9)}$ & $C_{16}^{(12)}$ & $C_{16}^{(10)}$ & $C_{16}^{(5)}$ & $C_{16}^{(3)}$ & $C_{16}^{(14)}$ & $C_{16}^{(7)}$ & $C_{16}^{(11)}$ & $C_{16}^{(13)}$ & $C_{16}^{(8)}$ & $C_{16}^{(4)}$ & $C_{16}^{(2)}$ \\
\hline
\end{tabular}

Frequency domain spreading codes

\begin{tabular}{|c|c|c|c|c|c|c|c|c|c|c|c|c|c|c|c|c|}
\hline $\begin{array}{l}\text { Assignment } \\
\text { order }\end{array}$ & 1 & 2 & 3 & 4 & 5 & 6 & 7 & 8 & 9 & 10 & 11 & 12 & 13 & 14 & 15 & 16 \\
\hline$N_{F}=4$ & $C_{4}^{(0)}$ & $C_{4}^{(2)}$ & $C_{4}^{(3)}$ & $C_{4}^{(1)}$ & & & & & & & & & & & & \\
\hline$N_{F}=8$ & $C_{8}^{(0)}$ & $C_{8}^{(4)}$ & $C_{8}^{(6)}$ & $C_{8}^{(2)}$ & $C_{8}^{(5)}$ & $C_{8}^{(3)}$ & $C_{8}^{(7)}$ & $C_{8}^{(1)}$ & & & & & & & & \\
\hline$N_{F}=16$ & $C_{16}^{(0)}$ & $C_{16}^{(8)}$ & $C_{16}^{(12)}$ & $C_{16}^{(4)}$ & $C_{16}^{(10)}$ & $C_{16}^{(6)}$ & $C_{16}^{(14)}$ & $C_{16}^{(2)}$ & $C_{16}^{(9)}$ & $C_{16}^{(5)}$ & $C_{16}^{(13)}$ & $C_{16}^{(3)}$ & $C_{16}^{(11)}$ & $C_{16}^{(7)}$ & $C_{16}^{(15)}$ & $C_{16}^{(1)}$ \\
\hline
\end{tabular}

Table 2. Nonsequential code assignment schemes.

explained before, for any data code channels, the MCI-F caused by other code channels in $\Omega_{F}$ is anticipated to be much higher than the MCI-T caused by code channels in $\Omega_{T}$. Therefore, at the receiver, the $2 \mathrm{D}$ despreader employs a simple EGC to collect spread signals in the time domain, while a promising detection technique, called hybrid MCI cancellation and minimum mean square error (MMSE) detection (hybrid detection), has been proposed for signal detection in the frequency domain to cancel out the MCI-F [5].

Assume a fully loaded OFCDM system with a $2 \mathrm{D}$ spreading factor of $N=4 \times 2$. Consider the code channel with time domain spreading code $\{+1,-1,+1,-1\}$ and frequency domain spreading code $\{+1,-1\}$. Then its subset $\Omega_{T}$ includes all other code channels with different time domain spreading codes such as $\{+1,+1$, $+1,+1\},\{+1,+1,-1,-1\}$, and $\{+1,-1,-1$, $+1\}$, while the subset $\Omega_{T}$ has one code channel with the same time domain spreading code $\{+1,-1,+1,-1\}$ and a different frequency domain spreading code $\{+1,+1\}$. The $2 \mathrm{D}$ despreading of the concerned code channel is illustrated in Fig. 3, which is carried out over the received signals on two interleaved subcarriers in four continuous OFCDM symbol durations. Note that each of the received signals is a combination of different signals from multiple code channels. To recover the information delivered by the desired code channel, time domain despreading is first carried out on each subcarrier, where the signals are multiplied by the EGC weights (or channel phases) and time domain spreading code $\{+1,-1,+1,-1\}$, and then summed over $N_{T}$ chips. In a slow fading channel the concerned code channel and all channels in its $\Omega_{T}$ keep orthogonal to each other. Thus, after time domain despreading, the signals transmitted on channels in $\Omega_{T}$ are eliminated from the multicode signal. But the resultant signal is still a combined one, including the desired signal and those transmitted on the channels in $\Omega_{F}$.
The outputs of time domain despreading on the $N_{F}$ interleaved subcarriers carrying the same data symbol are then processed in the frequency domain with hybrid detection. The basic idea of hybrid detection is shown in Fig. 3. At the 0th stage, the signals at the output of the time domain despreader with the EGC are multiplied by the MMSE weights and frequency domain spreading code $\{+1,-1\}$ and summed over $N_{F}$ chips, that is, the 0 th stage of the hybrid detection is conventional MMSE (or pure MMSE) combining without MCI cancellation. In this way, the desired signal is recovered with the inference from $\Omega_{F}$ suppressed by MMSE detection. Then tentative data decisions are obtained from the demodulation output in uncoded systems, or the decoding output in coded systems. At the 0th stage, tentative decisions from code channels belonging to $\Omega_{F}$ are input to a MCI regenerator with channel information (estimated by using the pilot channel) so that the MCI-F can be regenerated for the concerned code channel. At the first stage of the hybrid detection, the regenerated MCI-F from the 0th stage is fed back and canceled out from the output of the time domain despreader. The subtracted outputs are then multiplied by weights obtained from MMSE algorithms and combined at the frequency domain despreader to get the signals of a desired code channel. Since the MMSE weights are related to the input signal power, it should be updated stage by stage due to the reduction of MCI at each stage. A detailed description on renewing the MMSE weights in hybrid detection can be found in [5].

After the MCI cancellation and MMSE detection at the first stage, new data decisions are obtained. Similarly, they are used to regenerate the MCI-F for MCI cancellation at the next stage. This iterative operation will go on until a specified number of stages is reached. Generally speaking, as the tentative data decisions become more reliable stage by stage, MCI-F can be regenerated with higher accuracy. After subtrac- 


Using the Gaussian-
softdecision function,
the system
performance should
be improved
substantially in a
highly frequency
selective channel,
compared to that
using hard decision
function.

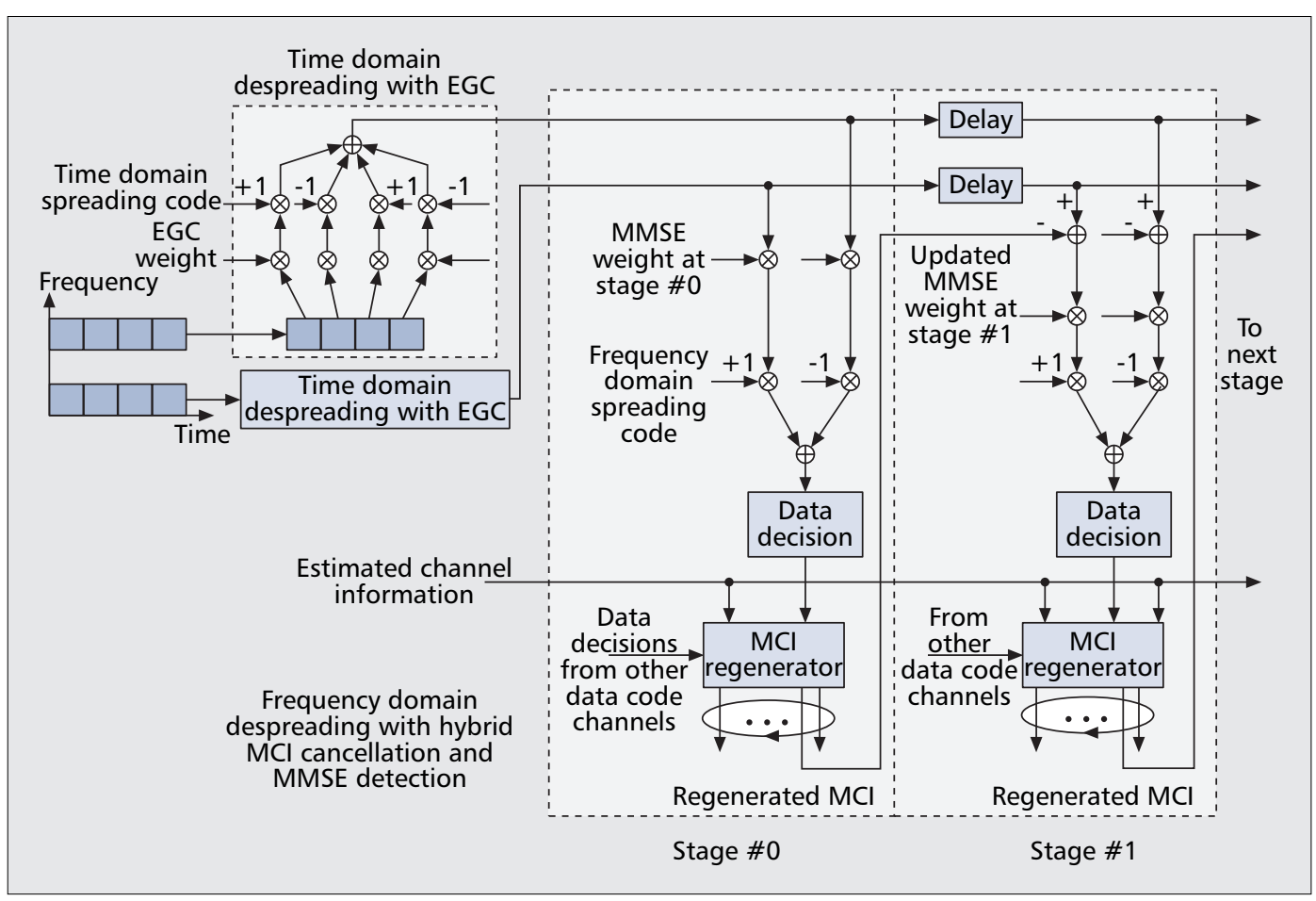

Figure 3. Example of $2 D$ despreading with $\mathrm{N}=\mathrm{N}_{\mathrm{T}} \times \mathrm{N}_{\mathrm{F}}=4 \times 2$. tion, much of the MCI-F can be canceled out from the received signal. Thus, the system performance can be improved stage by stage. Apparently, hybrid detection is more complex than pure MMSE without MCI cancellation, but the complexity increases only linearly with the number of stages. Hybrid detection is a good compromise between performance and complexity, and is an excellent detection technique for an OFCDM system.

When hybrid detection is employed in a turbo-coded OFCDM system [8], special considerations should be taken on the turbo decoder since the tentative data decisions used to regenerate $\mathrm{MCI}$ are obtained after turbo decoding. In a conventional turbo decoder, only the information bits (or systematic bits) are concerned, and the decoding algorithm derives the logarithm of likelihood ratio (LLR) of each systematic bit. As a result, only systematic bits are recovered after turbo decoding. However, in the interference cancellation of hybrid detection, a data replica must be regenerated, which requires recovering all coded bits. There are two methods to recover the parity bits. One method is to encode the recovered systematic bits to generate the parity bits. This method is simple, but the errors in systematic bits will propagate to parity bits due to the recursive encoder. Another method is to recover parity bits directly from turbo decoding. Based on the decoding algorithm used for deriving the LLR of systematic bits, the LLR of each parity bit can be obtained in a similar way [8]. Because of no error propagation, the latter method provides better performance than the former. Therefore, in hybrid detection, the calculation of LLR for parity bits should be added in the conventional turbo decoding and this extended algorithm will recover both systematic and parity bits.

\section{HARD VS. SOFT DECISIONS}

As stated before, the basic idea of hybrid detection is to use the decision signals of a previous stage to regenerate MCI, then subtract MCI from the input signal. The decision function could be hard or soft. The hard decision function is easy to realize, and MCI can be totally eliminated if the tentative decisions of the previous stage are correct. However, when the tentative decisions are wrong, MCI will be increased considerably by error propagation. In order to cancel out MCI as much as possible, a soft decision function should be used to regenerate MCI. The soft MCI cancellation approach is more complex, but can provide better performance than the hard decision one by alleviating the effect of error propagation.

In a turbo-coded OFCDM system with soft MCI cancellation, the turbo decoding output must pass through a soft decision function to generate soft tentative decisions. The soft decision function can be designed by minimizing the mean square decision error. The derivation based on this criterion reveals that the soft decision function is determined by the statistic property of input variable (i.e., the turbo decoding output) [9]. Assuming that the turbo decoding output can be approximated as a binary phase shift keying (BPSK) modulated signal passing through an AWGN channel, Gaussian soft decision can be derived. Using the Gaussian softdecision function, system performance should be improved substantially in a highly frequency selective channel over that using a hard decision function.

\section{MIMO-OFCDM}

Similar to OFDM, OFCDM readily supports MIMO antenna techniques. Aiming to provide extra high data rates, OFCDM employs spatial 


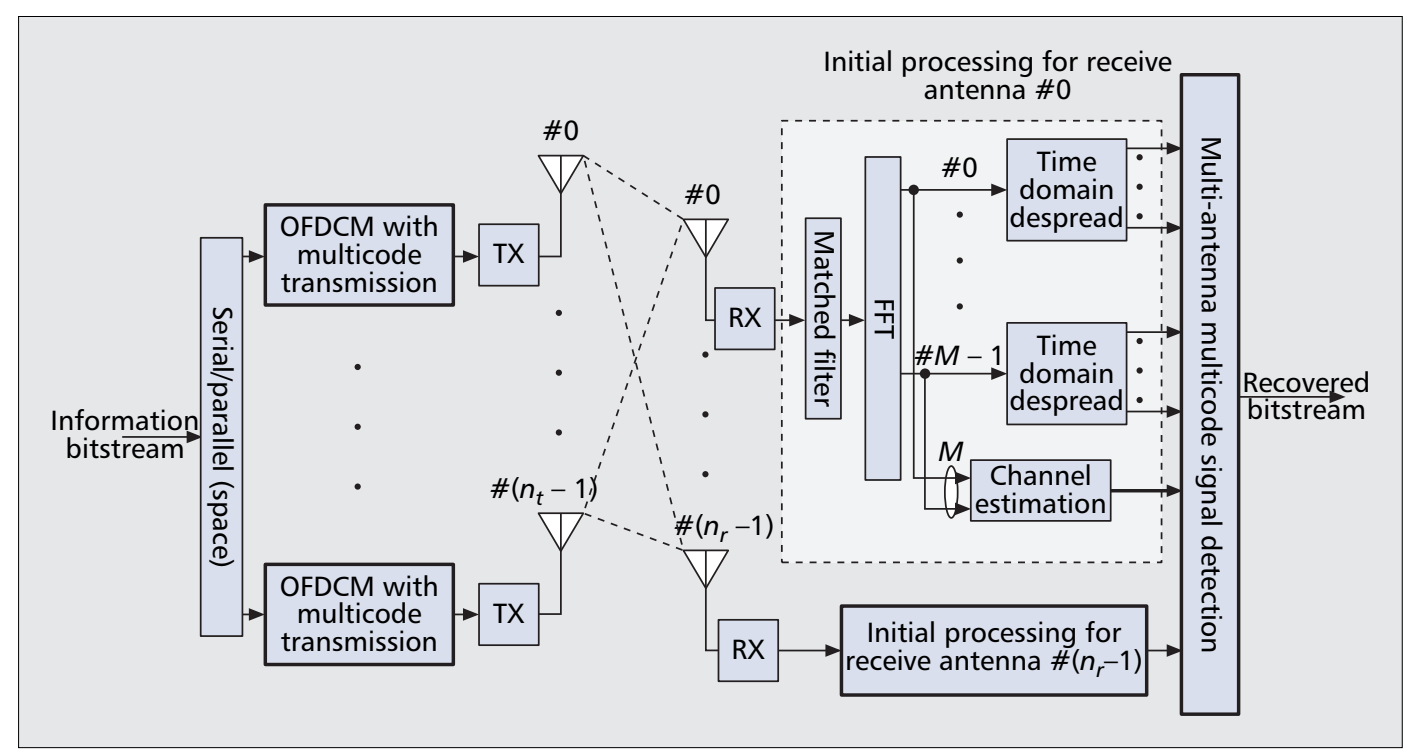

Figure 4. MIMO-OFCDM system model.

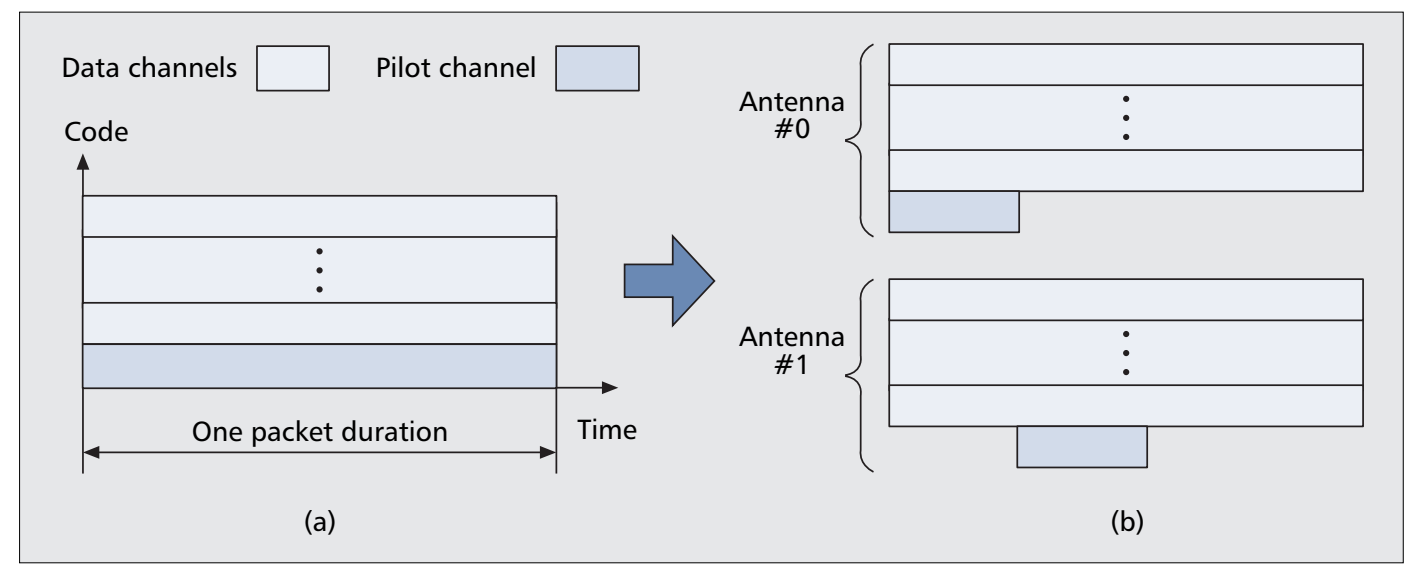

Figure 5. Illustration of pilot structure in a) OFCDM; b) MIMO-OFCDM.

domain multiplexing or MIMO multiplexing, where different data symbols are transmitted on different antennas simultaneously, so the data rate can be increased linearly with the minimum number of transmit and receive antennas. The system model of MIMO-OFCDM is shown in Fig. 4. At the transmit side, information bitstreams are first serial to parallel $(\mathrm{S} / \mathrm{P})$ converted into $n_{t}$ substreams, corresponding to $n_{t}$ transmit antennas. Each substream is fed to the 2D spread OFCDM with multicode transmission, which will produce OFCDM symbols. The process of the 2D spread OFCDM is similar to the one in Fig. 2. However, a small modification is needed on the pilot channels. As illustrated in Fig. 5, in the single antenna OFCDM system, the code multiplexed pilot channel exists through the entire packet duration. However, to obtain good channel estimation in the MIMO-OFCDM system, the same time domain spreading code is used for all the pilot channels, and the pilot symbols are sent on different antennas in different time slots. Using this pilot structure, each pilot channel can keep orthogonal to all transmitted data channels as well as other pilot channels.

At the receive side, $n_{r}$ antennas are employed.
As shown in Fig. 4, on each receive antenna, the initial processing is similar to that in a single antenna OFCDM system (Fig. 2). The radio signal is processed by the matched filter and an FFT block. On each subcarrier, the time domain despreader is carried out with EGC to collect the useful signals in the time domain. The output of the time domain despreader is then input to a multi-antenna multicode signal detector with the estimated channel information. As stated before, in a broadband mobile channel, MCI exists among the data channels in OFCDM systems when multicode transmission is considered, especially in the frequency domain. Therefore, in a MIMO-OFCDM system, desired data signals are interfered with by not only co-channel signals from other transmit antennas (or multiantenna interference [MAI]), but also MCI from other code channels. The multi-antenna multicode detection is proposed for the MIMOOFCDM system to deal with MAI as well as MCI.

Given $n_{t}=n_{r}=4$, the signal processing in multi-antenna multicode detection is illustrated in Fig. 6. Since the power of MAI in the space domain could be much larger than the power of
The same time domain spreading code is used for all the pilot channels,

and the pilot

symbols are sent on

different antennas in

different time slots.

Using this pilot

structure, each pilot channel can keep orthogonal to all transmitted data channels as well as other pilot channels. 


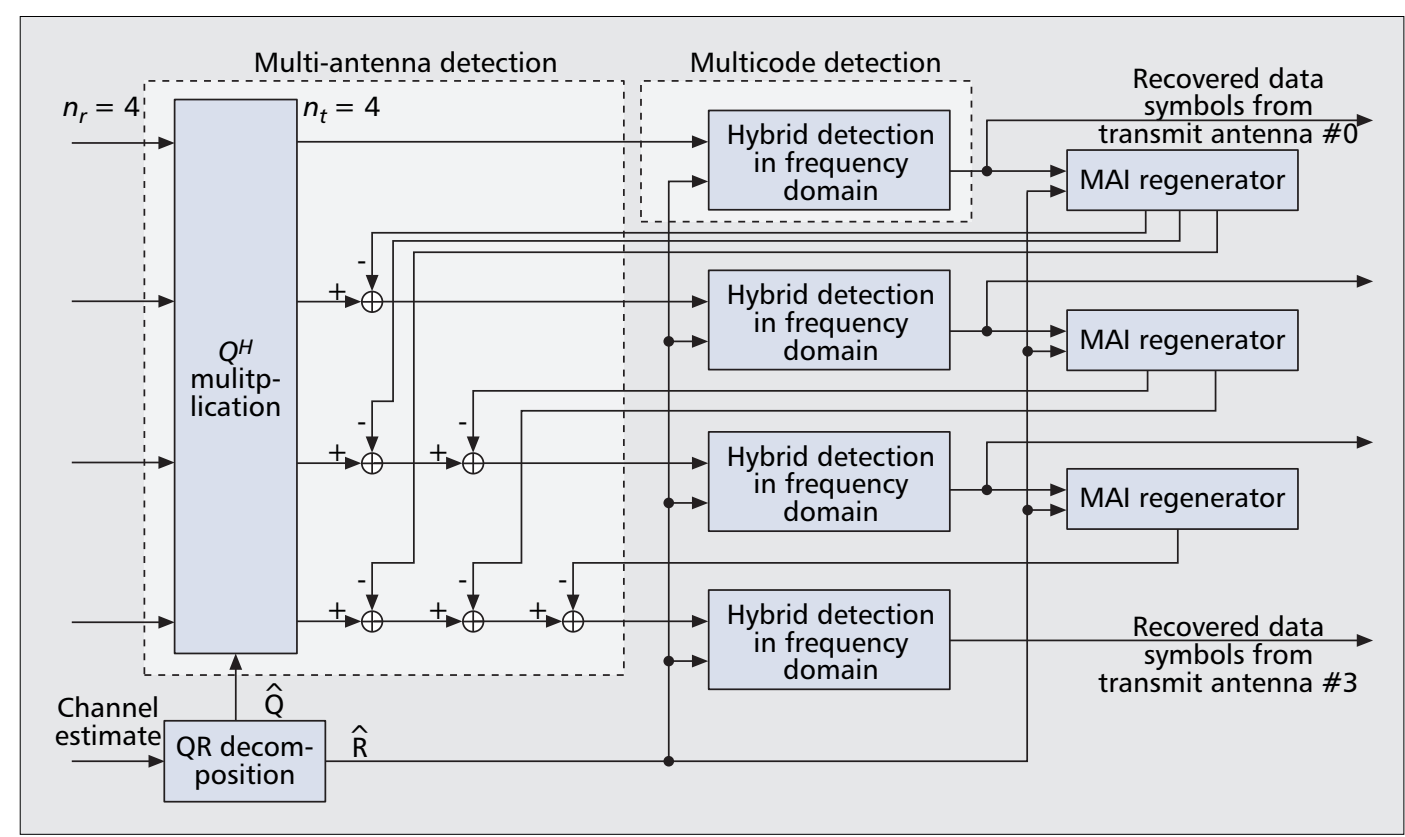

Figure 6. Example of multi-antenna multicode signal detection for $\mathrm{n}_{\mathrm{t}}=\mathrm{n}_{\mathrm{r}}=4$.

\begin{tabular}{|c|c|c|c|c|}
\hline Functions & OFDM & \multicolumn{3}{|c|}{ OFCDM } \\
\hline $\begin{array}{l}\text { 2D } \\
\text { spreading }\end{array}$ & No & \multicolumn{3}{|c|}{$\left[\left(N_{T}-1\right) N_{F}-1\right] M \cdot N_{d}$ additions } \\
\hline \multirow{4}{*}{$\begin{array}{l}\text { 2-D } \\
\text { despreading }\end{array}$} & \multirow{4}{*}{ No } & \multirow{2}{*}{\multicolumn{2}{|c|}{ EGC combining }} & $\begin{array}{l}\left(N_{T}-1\right)^{2} M \cdot N_{d} / N_{T} \\
\text { additions }\end{array}$ \\
\hline & & & & $\begin{array}{l}\left(N_{T}-1\right) M \cdot N_{d} \\
\text { multiplications }\end{array}$ \\
\hline & & \multirow{2}{*}{$\begin{array}{l}\text { Hybrid } \\
\text { detection }\end{array}$} & $\begin{array}{l}\text { MMSE } \\
\text { detection }\end{array}$ & $\begin{array}{l}\left(N_{F}-1\right) M \cdot N_{d}(s+1) \\
\text { additions } \\
N_{F} M \cdot N_{d}(s+1) \\
\text { multiplications }\end{array}$ \\
\hline & & & $\begin{array}{l}\mathrm{MCl} \\
\text { regeneration }\end{array}$ & $\begin{array}{l}\left(N_{T}-1\right) N_{F} M \cdot N_{d} \cdot s \\
\text { additions } \\
\left(N_{T}-1\right) N_{F} M \cdot N_{d} \cdot s \\
\text { multiplications }\end{array}$ \\
\hline $\begin{array}{l}\text { Turbo } \\
\text { decoding }\end{array}$ & Once & \multicolumn{3}{|c|}{$(s+1)$ times } \\
\hline
\end{tabular}

Table 3. Complexity comparison between OFDM and OFCDM.

MCI in the code domain, the elimination of MAI is considered first, which is realized by using a zero-forcing MAI nulling and cancellation based on QR decomposition (ZF-QRD) in the space domain [10]. It is assumed that $\hat{\mathbf{H}}_{m}$ is a $n_{t} \times n_{r}$ estimated channel matrix with elements $\hat{h}_{m, i, j}$ standing for the fading factor on the $m$ th subcarrier when the signal is transmitted from the $i$ th antenna and received by the $j$ th antenna. The QR decomposition of $\hat{\mathbf{H}}_{m}$ is given by $\hat{\mathbf{H}}_{m}=$ $\hat{R}_{m} \hat{Q}_{m}$, where $\hat{R}_{m}$ is an $n_{t} \times n_{r}$ upper triangular matrix and $\hat{Q}_{m}$ is an $n_{t} \times n_{r}$ orthogonal matrix.
By multiplying the received signals on the $n_{r}$ antennas with the hermitian transpose of $\hat{Q}_{m}$ (i.e., $\left.\hat{Q}_{m}^{H}\right), n_{t}$ signals can be obtained, which contain desired signals from $n_{t}$ transmitting antennas. Although there is no MAI in the signal from the 0th antenna, the obtained signal from the $p$ th $(p=1,2,3)$ transmitting antenna is contaminated by MAI from the 0 th $-(p-1)$ th antennas. Consider the signal from the 0th transmitting antenna first. Without MAI from other antennas, the signal is still a composite multicode signal. Therefore, hybrid MCI cancellation and MMSE detection is carried out to recover the data symbols on each code channel. When the data symbols are obtained, MAI caused by the 0 th antenna can be regenerated and subtracted from the outputs of $Q^{H}$ multiplication. Since the signal from the first transmitting antenna obtained after $Q^{H}$ multiplication is interfered with by MAI from only the 0th antenna, after MAI cancellation, the signal can be assumed to be free of MAI and further processed by the hybrid detection. Similarly, when the data symbols on the first transmitting antenna are recovered after hybrid detection, MAI caused by the first tranmitting antenna is regenerated and subtracted from the outputs of $Q^{H}$ multiplication. This recursive process will go on until the data symbols from the $\left(n_{t}-1\right)$ th antenna are recovered. It has been shown that using this multiantenna multicode detection, the MIMO-OFCDM system with 2D spreading outperforms the conventional MIMO-OFDM system without spreading.

\section{COMPLEXITY OVERHEAD}

As can be seen from previous sections, the complexity of the OFCDM system is higher than that of the OFDM system due to the introduction of 2D spreading and despreading. Assuming singleantenna systems, a complexity comparison between OFCDM and OFDM is shown in Table 
3 , where $N_{d}$ is the number of OFCDM symbols in one packet duration, $M$ is the total number of subcarriers, and an $S$-stage hybrid detection is employed in OFCDM. It can be seen that the operation of 2D spreading and EGC combining with time domain despreading is relatively simple. The complexity introduced by 2D spreading and EGC combining is determined by system parameters $N_{d}, M, N_{T}$, and $N_{F}$. However, for hybrid detection, which includes MMSE detection and MCI regeneration, the complexity also increases with the number of stages in hybrid detection $s$. Moreover, when a turbo-coded system is considered, OFDM only needs one turbo decoding operation, while for OFCDM, $(s+1)$ times of turbo decoding are necessary since the decoding is included in hybrid detection. Therefore, the complexity of OFCDM is mainly due to the multistage hybrid detection. Fortunately, the complexity only increases linearly with s and other system parameters. With the development of microelectronics, the complexity in basedband processing is not difficult to handle.

\section{SiMULATION RESULTS}

Some representative computer simulation results are presented to show the advantage of OFCDM over OFDM in various circumstances. The downlink transmission in a single cell environment is considered. Assuming scheduling in downlink, there is no multi-user interference. Thus, in the OFDM system, data signals are corrupted by background noise but no other interference. However, in the OFCDM system, although there is no intercell or multi-user interference, MCI is unavoidable due to multicode transmission. The OFCDM system is configured as follows. A 2/3 rate turbo code and QPSK modulation are employed. The time domain spreading factor $N_{T}$ is fixed to 8 , while the frequency domain spreading factor $N_{F}$ can be varied. A fully loaded system is assumed where all $\left(N_{T}-1\right) N_{F} 2 \mathrm{D}$ code channels are used for data transmission. As for the OFDM system, similar system configurations are applied except that a time-multiplexed pilot channel with $N_{P}=6$ is employed. Due to no spreading, conventional correlators are used for OFDM. Using the system parameters assumed here and in Table 1, the maximum transmission rate of either turbo-coded OFCDM or turbocoded OFDM is $95 \mathrm{Mb} / \mathrm{s}$. As to the channel model, although the broadband channel is highly frequency selective, the signal transmitted on each subcarrier experiences a flat fading channel. Therefore, a parallel slow and flat fading multichannel model with a coherence bandwidth $\Delta f_{c}=0.47 \mathrm{MHz}$ is assumed.

First of all, the bit error rate (BER) performance of an uncoded OFCDM is shown in Fig. 7 for $N=N_{T} \times N_{F}=8 \times 16$. A three-stage hybrid detector, including the 0th to third stages, is considered. The BER of the unspread OFDM system is also plotted as a reference. Since the OFCDM obtains frequency diversity gain by means of frequency domain spreading, OFCDM with 2D spreading provides much better performance than OFDM, even if pure MMSE detection (or the 0th stage) is employed. The performance enhancement of OFCDM over

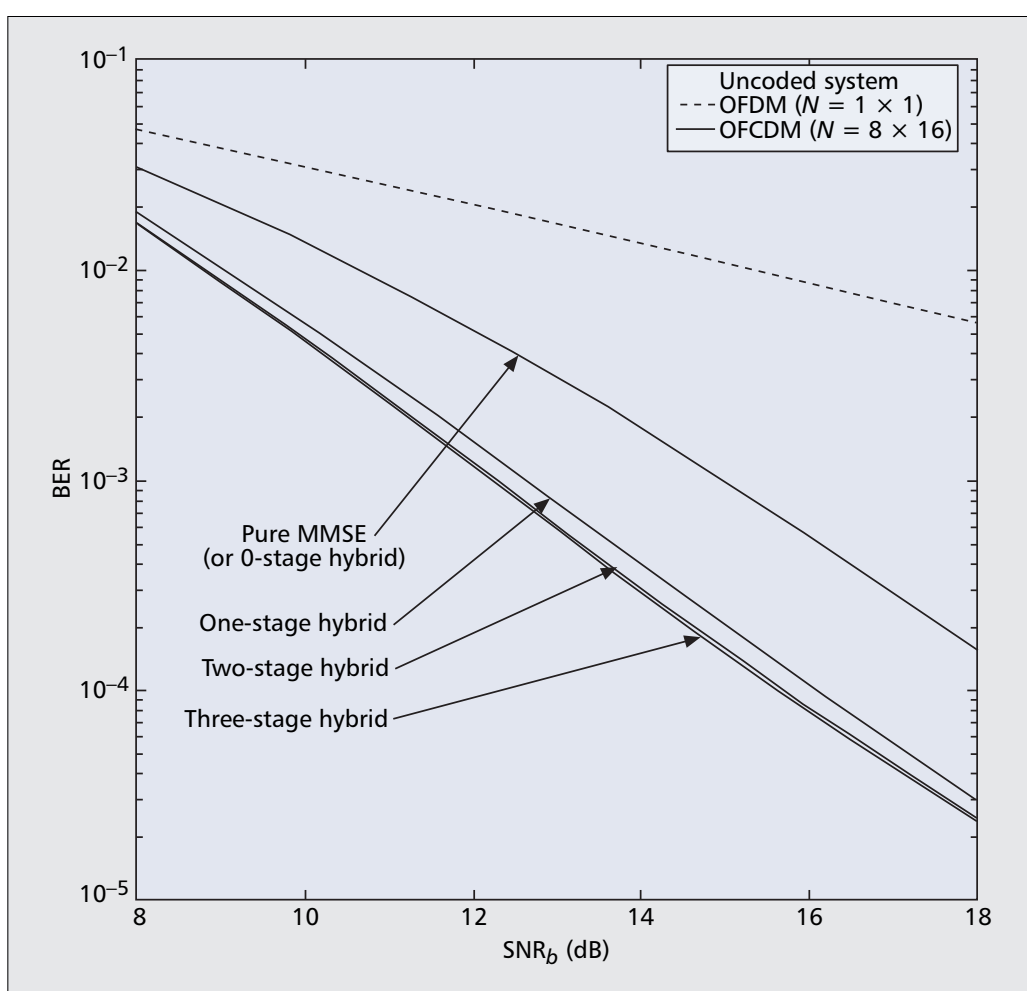

Figure 7. Performance comparison between uncoded OFCDM and OFDM systems.

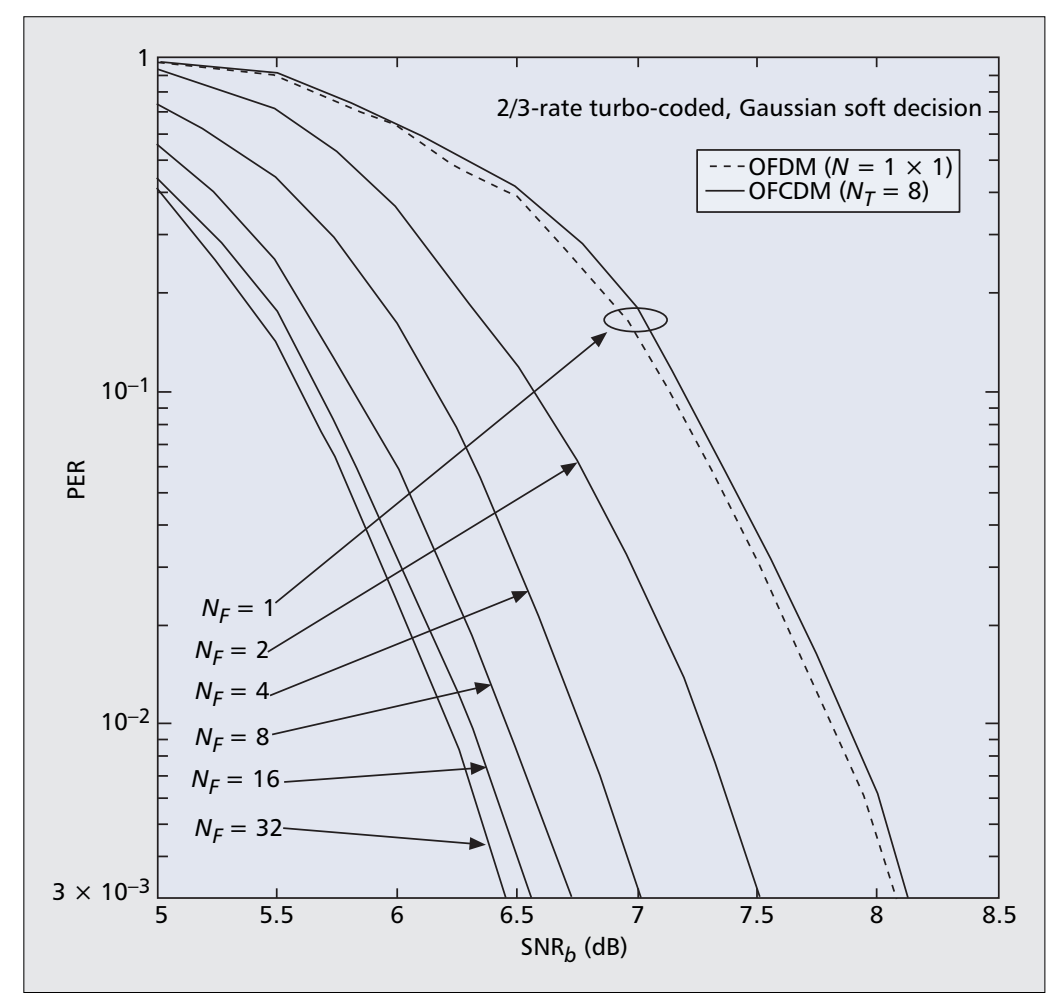

Figure 8. Performance of turbo-coded OFCDM and OFDM systems.

OFDM increases with signal-to-noise ratio (SNR). Moreover, using hybrid detection, the BER of OFCDM can be further improved as the number of stages increases. The performance improvement between pure MMSE (or 0th stage) and hybrid detection with the first stage is 
significant. The gap in BER between the first and second stages is also big. However, the improvement beyond the second stage is insignificant. Therefore, the BER performance improvement for hybrid detection decreases as the stages increase. In conclusion, when an uncoded system is considered, the $2 \mathrm{D}$ spread OFCDM with

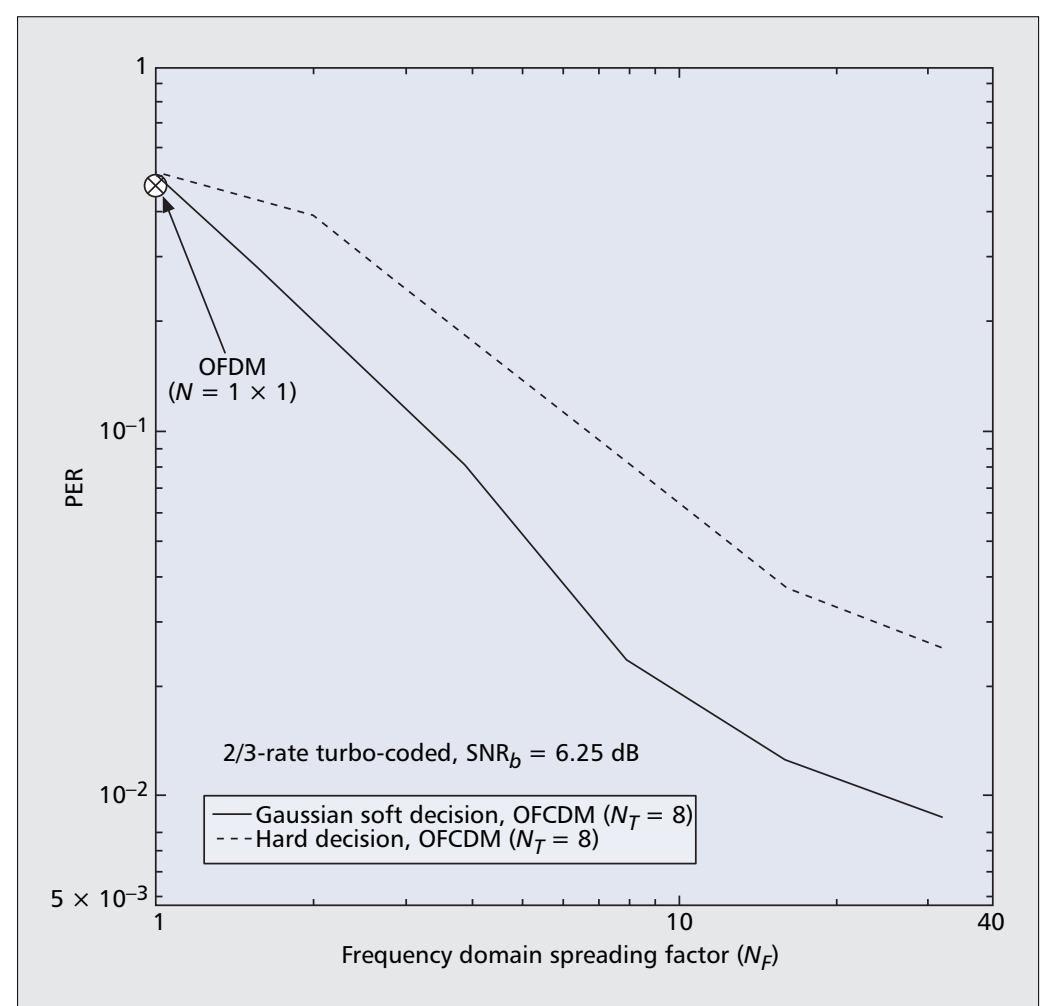

Figure 9. Turbo-coded OFCDM with soft and hard MCI cancellation.

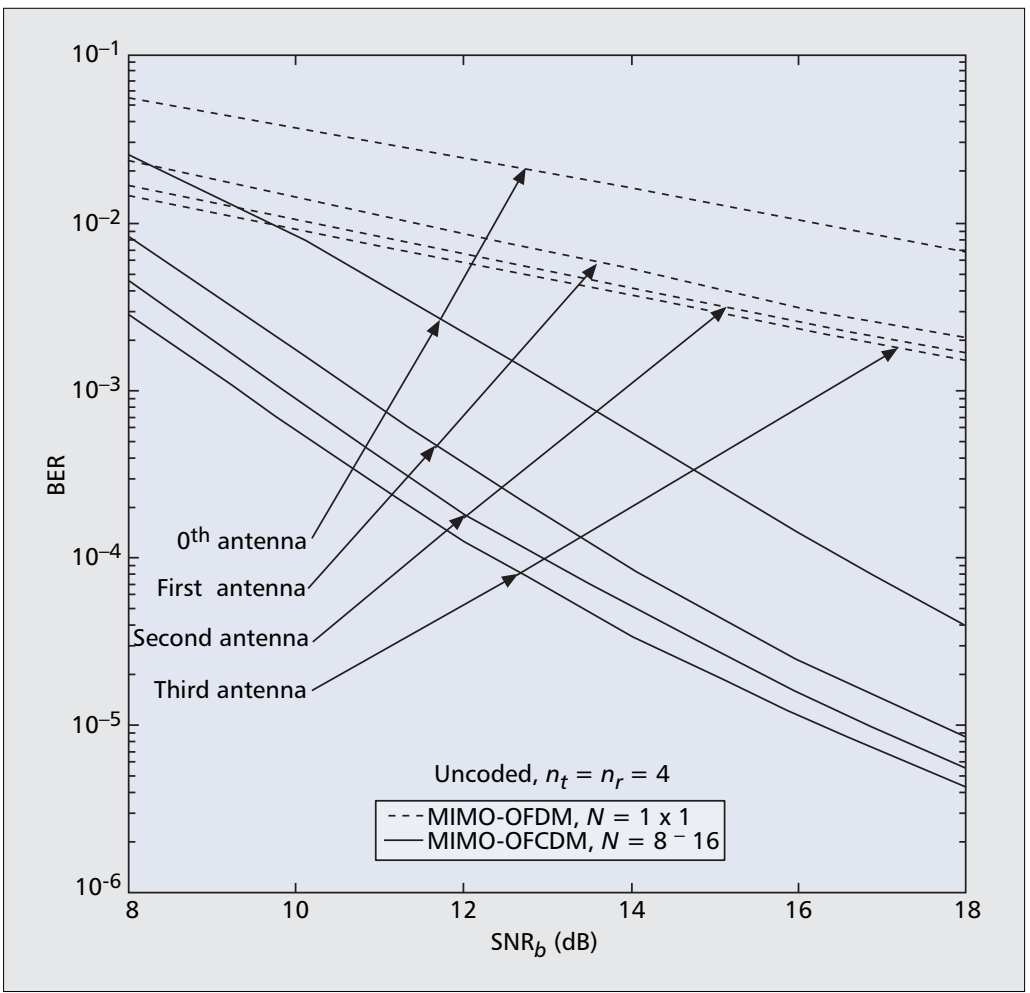

Figure 10. Comparison between MIMO-OFCDM and MIMO-OFDM. hybrid detection outperforms the unspread OFDM, especially when SNR is large.

When turbo coding is considered, the PER performance comparison is carried out in Fig. 8 between the OFCDM and OFDM as a function of SNR. In the OFCDM system, when $N_{F}$ is one, there is no need for both frequency domain despreading and hybrid detection. When $N_{F} \geq 2$, seven-stage soft MCI cancellation and MMSE detection is employed with the Gaussian soft decision function [9]. It can be seen that OFDM has almost the same performance as OFCDM with $N_{F}=1$. Given $N_{F}=1$, OFCDM only employs time domain spreading. Under the assumption of slow fading, the orthogonality between code channels can be maintained at the receiver. Thus, time domain spreading does not cause MCI. Therefore, OFCDM with $N_{F}=1$ has similar performance to that of OFDM. However, when $N_{F}$ increases, the performance of the OFCDM system is enhanced significantly and surpasses that of OFDM, especially at high SNR. Although turbo-coded OFDM can also obtain frequency diversity gain due to channel coding, turbo-coded OFCDM achieves even larger gain because of both channel coding and frequency domain spreading. Moreover, for OFCDM, the frequency diversity gain increases with $N_{F}$. Although more MCI is caused when $N_{F}$ gets larger, soft MCI cancellation can suppress MCI greatly. In this case frequency diversity can still provide more gain when $N_{F}$ becomes larger. However, the performance improvement gets smaller as $N_{F}$ increases further; finally, when $N_{F}$ is larger than 16, the frequency diversity gain is gradually saturated, and the improvement becomes insignificant. In summary, when turbo coding is considered, the OFCDM system has a big advantage over the OFDM system in system performance.

Moreover, Fig. 9 shows the performance comparison between OFCDM with soft and hard MCI cancellation. Given $\mathrm{SNR}_{b}=6.25 \mathrm{~dB}$, the performance is plotted as a function of the frequency domain spreading factor, $N_{F}$. For comparison, the packet error rate (PER) of OFDM is also shown in the figure, which almost coincides with that of OFCDM with $N_{F}=1$. It can be seen that the Gaussian soft decision function outperforms the hard decision function for all values of $N_{F}$ except $N_{F}=1$. As $N_{F}$ increases, the performance of the OFCDM system improves significantly. This is consistent with Fig. 8. Again, turbo-coded OFCDM is superior to turbo-coded OFDM.

Finally, assuming four transmit and four receive antennas, the performance of MIMOOFCDM is illustrated in Fig. 10 as a function of SNR. Given a 2D spreading scheme of $N=N_{T}$ $\times N_{F}=8 \times 16$, two-stage hybrid detection is used in the multi-antenna multicode detection of MIMO-OFCDM. For both the MIMO-OFCDM and MIMO-OFDM systems, the BER of the data symbol on each transmitting antenna is shown. It can be seen that MIMO-OFCDM outperforms MIMO-OFDM. With $N_{F}=16$ and hybrid detection, MIMO-OFCDM benefits from frequency diversity gain. Thus, the BER on each antenna is considerably reduced in the MIMOOFCDM system from that in the MIMO-OFDM 
system. The higher the SNR, the larger the performance improvement obtained by MIMOOFCDM over MIMO-OFDM. Hence, OFCDM outperforms OFDM when the MIMO technique is applied.

\section{CONCLUSIONS}

In order to meet future high traffic expectations, high-speed data transmission is needed in future wireless systems, especially in the downlink. OFCDM has become a very attractive wireless access technique for future $4 \mathrm{G}$ mobile communications due to its superiority to OFDM.

\section{REFERENCES}

[1] T. Ikeda et al., "Experimental Evaluation of Coherent Rake Combining for Broadband Single-Carrier DS-CDMA Wireless Communications," IEICE Trans. Commun., vol. E84-B, no. 3, Mar. 2001, pp. 415-24.

[2] H. Atarashi, S. Abeta, and M. Sawahashi, "Broadband Packet Wireless Access Appropriate for High-Speed and High-Capacity Throughput," IEEE VTC '01-Spring, May 2001, pp. 566-70.

[3] Y. Kishiyama et al., "Transmission Performance Analysis of VSF-OFCDM Broadband Packet Wireless Access based on Field Experiments in 100-MHz Forward Link," Proc. IEEE VTC '04-Fall, vol. 5, Sept. 2004, pp. 3328-33.

[4] F. Adachi, M. Sawahashi, and H. Suda, "Wideband DSCDMA for Next-Generation Mobile Communications Systems," IEEE Commun. Mag., vol. 36, no. 9, Sept. 1998, pp. 56-69.

[5] Y. Q. Zhou, J. Wang, and M. Sawahashi, "Downlink Transmission of Broadband OFCDM Systems - Part I: Hybrid Detection," IEEE Trans. Commun., vol. 53, Apr. 2005, pp. 718-29.

[6] K. Okawa et al., "1.92 Mb/s Data Transmission Experiments over a Coherent W-CDMA Radio Link," IEICE Trans. Commun., vol. E81-B, no. 7, July 1998, pp. 1330-36.

[7] Y. Q. Zhou, J. Wang, and T. S. Ng, "Downlink Transmis sion of Broadband OFCDM Systems - Part V: Code Assignment," to appear, IEEE Trans. Wireless Commun.

[8] Y. Q. Zhou, J. Wang, and M. Sawahashi, "Downlink Transmission of Broadband OFCDM Systems - Part III: Turbocoded," IEEE JSAC, vol. 24, Jan. 2006, pp. 132-40.

[9] Y. Q. Zhou and J. Wang, "Downlink Transmission of Broadband OFCDM Systems -Part IV: Soft Decision," IEEE JSAC, vol. 24, June 2006, pp. 1208-20.

[10] K. Higuchi et al., "Adaptive Selection of Surviving Symbol Replica Candidates based on Maximum Reliability in QRM-MLD for OFCDM MIMO Multiplexing," IEEE GLOBECOM '04, vol. 4, 2004, pp. 2480-86.

\section{BIOGRAPHIES}

YIQING ZHOU [S'03, M'05] received a B.S. degree in communication and information engineering, and an M.S. degree in signal and information processing from Southeast University, China, in 1997 and 2000, respectively. In February 2004 she received a Ph.D. degree in electrical and electronic engineering from the University of Hong Kong. Since June 2004 she has been with the Department of Electrical and Electronic Engineering at the University of Hong Kong as a postdoctoral fellow. Her research interests include coding theory, spread spectrum, OFDM systems, interference cancellation, hybrid ARQ, MIMO, and other transmission techniques for wireless high-speed data communications.

JIANGZHOU WANG [M'91, SM'94] received B.S. and M.S. degrees from Xidian University, Xian, China, in 1983 and 1985, respectively, and a Ph.D. degree (with Greatest Distinction) from the University of Ghent, Belgium, in 1990, all in electrical engineering. He is currently a professor and chair of the Department of Electronics, University of Kent United Kingdom. From 1995 to 2005 he was with the University of Hong Kong, where he is still serving as an honorary professor. From 1992 to 1995 he was a senior system engineer at Rockwell International Corporation (now Conexant), California. From 1990 to 1992 he was a postdoctoral fellow at the University of California at San Diego. He has served as a visiting professor at NTT DoCoMo, Japan. He was a Technical Chairman of the IEEE Workshop in 3G Mobile Communications in 2000. He has been a technical committee member and session chair of a number of international conferences. He has published over 140 papers, including more than 40 IEEE transactions/journal papers in the areas of wireless mobile and spread spectrum communications. He has written/edited three books: High Speed Wireless Communications (Cambridge University Press, 2008), Broadband Wireless Communications (Kluwer, 2001), and Advances in 3 G Enhanced Technologies for Wireless Communications (Artech House, 2002) respectively. The last book has been translated into Chinese. He is an Editor for IEEE Transactions on Communications and has been a Guest Editor of IEEE Journal on Selected Areas in Communications three times. He holds one U.S. patent on the GSM system. He is an Evaluation Expert of the European Commission Framework Program 7 (FP7). He is listed in Who's Who in the World.

TUNG-SANG NG [F] received a B.Sc.(Eng.) degree from the University of Hong Kong in 1972, and M.Eng.Sc. and Ph.D. degrees from the University of Newcastle, Australia, in 1974 and 1977, respectively, all in electrical engineering. He worked for BHP Steel International and the University of Wollongong, Australia, after graduation for 14 years before returning to the University of Hong Kong in 1991, taking up the position of professor and chair of Electronic Engineering. He was head of the Department of Electrical and Electronic Engineering from 2000 to 2003 and dean of engineering from 2003 to 2007 . His current research interests include wireless communication systems, spread spectrum techniques, CDMA, and digital signal processing. $\mathrm{He}$ has published over 250 international journal and conference papers. He was General Chair of ISCAS '97 and VPRegion 10 of IEEE CAS Society in 1999 and 2000. He was an Executive Committee Member and a Board Member of the IEE Informatics Divisional Board (1999-2001) and an ordinary member of IEE Council (1999-2001). He was awarded the Honorary Doctor of Engineering Degree by the University of Newcastle, Australia, in 1997, the Senior Croucher Foundation Fellowship in 1999, the IEEE Third Millenium medal in 2000, and the Outstanding Researcher Award of the University of Hong Kong in 2003. He is a Fellow of IEE and HKIE.

KENICHI HIGUCHI (M'05) received a B.E. degree from Waseda University, Tokyo, Japan, in 1994, and received his Dr.Eng. degree from Tohoku University, Sendai, Japan, in 2002. In 1994 he joined NTT Mobile Communications Network, Inc. (now NTT DoCoMo, Inc.). Since joining NTT, he has been engaged in the research and development of wireless access technologies including code synchronization, interference cancellation, and multiple-antenna transmission techniques for wideband DS-CDMA mobile radio and broadband wireless packet access technologies for systems beyond IMT-2000. In 2007 he joined Tokyo University of Science. He is currently a junior associate professor at Tokyo University of Science. He is a member of the Institute of Electronics, Information, and Communication Engineers (IEICE) of Japan.

MAMORU SAWAHASHI (M'88) received his B.S. and M.S. degrees from Tokyo University in 1983 and 1985, respectively, and received his Dr. Eng. degree from the Nara Institute of Technology in 1998. In 1985 he joined the NTT Electrical Communications Laboratories, and in 1992 he transferred to NTT Mobile Communications Network, Inc. (now NTT DoCoMo, Inc.). In NTT he was engaged in the research of modulation/demodulation techniques for mobile radio. He was also engaged in the research and development of radio access technologies for W-CDMA mobile communications and broadband packet radio access technologies for $3 \mathrm{G}$ long-term evolution and the systems beyond IMT-2000 in NTT DoCoMo. In April 2006 he assumed the position of professor in the Department of Electronics and Communication Engineering, Musashi Institute of Technology. Since 2007 he has been a part-time director of the Radio Access Development Department of NTT DoCoMo. He is currently serving as an Editor for IEEE Transactions on Wireless Communications.
In order to meet

future high traffic

expectations,

high-speed data

transmission is

needed in future

wireless systems,

especially in the

downlink. OFCDM

has become a very

attractive wireless

access technique for

future $4 G$ mobile

communications

due to its superiority

to OFDM. 